\title{
Mutations of ESPN cause autosomal recessive deafness and vestibular dysfunction
}

\author{
S Naz, A J Griffith, S Riazuddin, L L Hampton, J F Battey Jr, S N Khan, S Riazuddin, E R Wilcox, \\ T B Friedman
}

\begin{abstract}
We mapped a human deafness locus DFNB36 to chromosome 1 p36.3 in two consanguineous families segregating recessively inherited deafness and vestibular areflexia. This phenotype co-segregates with either of two frameshift mutations, 1988delAGAG and 2469delGTCA, in ESPN, which encodes a calcium-insensitive actin-bundling protein called espin. A recessive mutation of ESPN is known to cause hearing loss and vestibular dysfunction in the jerker mouse. Our results establish espin as an essential protein for hearing and vestibular function in humans. The abnormal vestibular phenotype associated with ESPN mutations will be a useful clinical marker for refining the differential diagnosis of nonsyndromic deafness.
\end{abstract}

M ost congenital non-syndromic hearing loss is inherited as a recessive Mendelian disorder. ${ }^{1}$ Forty recessive deafness loci (DFNB) have been mapped and the genes responsible at 20 of these loci have been reported. ${ }^{2}$ With Institutional Review Board (IRB) approval and written informed consent we ascertained families PKSN32 and PKSR5A from Punjab, Pakistan. Detailed clinical histories were obtained for affected individuals (ages ranging from 6 to 40 years), who exhibited prelingual, profound, sensorineural hearing loss and independent ambulation delayed beyond 1.5 years of age. Affected members (ages 14, 16, and 39) of family PKSN32 denied vestibular symptoms although caloric testing with electronystagmography (ENG) revealed vestibular areflexia. The four deaf individuals in family PKSR5A reported balance problems, and caloric-ENG testing confirmed vestibular areflexia. Although congenital, profound hearing loss with vestibular areflexia is accompanied by retinitis pigmentosa (RP) in Usher syndrome type $1{ }^{3}$ affected individuals from PKSN32 or PKSR5A denied nyctalopia or other symptoms of RP. Electroretinography (ERG) on two affected individuals from families PKSN32 (age 14) and PKSR5A (age 19) ruled out the presence of RP.

The deafness segregating in family PKSN32 was not linked to any of the known recessive or dominant deafness loci (Hereditary Hearing Loss Homepage, http://nalabwww.uia.ac.be/dnalab/hhh/). Therefore, a genome-wide search was performed with the Marshfield Weber 9 marker set. We found a $6 \mathrm{cM}$ region of homozygosity on chromosome 1p36.3 among four affected individuals of this family (fig 1). A maximum two-point LOD score of $4.1(\theta=0)$ was obtained with marker D1S214, defining a new recessive deafness locus, DFNB36. In the second family, PKSR5A, the hearing loss was consistent with linkage to DFNB36 with a maximum two-point LOD score of $3.4(\theta=0)$ for marker D1S3774 (fig 1).

$E S P N$, a gene in the DFNB36 critical interval at 1p36.3, was a good positional candidate because a mutation of Espn is known to cause deafness and vestibular dysfunction in the jerker mouse. ${ }^{4}$ We screened the coding sequence of ESPN (GenBank accession number AL136880) by sequencing PCR amplified ESPN exons from genomic DNA of affected individuals in the two families. However, the sequencing analysis of ESPN was complicated due to the presence of a second ESPN-like sequence on chromosome 1p36.13, annotated as LOC284729 (GenBank accession number AL035288). As we identified frequent frameshift mutations in LOC284729 disrupting its open reading frame (data not shown) and due to the absence of sequence encoding homologous residues that are important for actin-bundling activity of espin, LOC284729 appears to be an unprocessed pseudogene. Based upon our analyses of LOC284729, this putative pseudogene has been assigned the symbol ESPNP. Except for the absence of sequences comparable to exon 1 and exon 13, ESPNP has all of the other exons and introns of ESPN to which it shows $95 \%$ nucleotide sequence identity. ESPNP is $10 \mathrm{Mb}$ away from $E S P N$ in reverse orientation with respect to ESPN and is outside the DFNB36 linkage interval defined by families PKSN32 (fig 1) and PKSR5A (data not shown).

Intronic primers for amplification of exons 1 and 13 were unique for ESPN since ESPNP lacks homologous sequences, and intronic primers for amplification of exons 2, 6, 7, 8, 9, and 10 were specific for ESPN due to multiple mismatches with ESPNP sequence (table 1). However, intronic primers for amplification of other exons were either identical to ESPN and ESPNP (exons 11 and 12) or failed to discriminate between the mismatches of the two sequences (exons 3, 4, and 5). Therefore, we subcloned the PCR products from exons $3,4,5,11$, and 12 and sequenced individual clones. The nucleotide mismatches allowed us to unambiguously assign individual clones to either ESPN or ESPNP.

We detected two different mutations of ESPN segregating with the deafness phenotype in families PKSR5A and PKSN32. In family PKSR5A, affected individuals were homozygous for a 4 bp deletion, 1988delAGAG, in exon 9 (fig 2A), while in family PKSN32 all affected individuals were homozygous for a 4 bp deletion, 2469delGTCA, in exon 13 (fig 2B). The obligate carriers in both families were heterozygous and neither mutation was detected among 150 normal-hearing individuals from Pakistan. The1988delAGAG mutation in exon 9 of ESPN was not present in ESPNP in deaf individuals of family PKSR5A. ESPNP lacks the site of 2469delGTCA mutation in family PKSN32 (fig 2C).

$E S P N$ is predicted to encode an 854 amino acid protein (fig 3 ) referred to as espin, a name derived from ectoplasmic specialisations $(+$ in) on the basis of its discovery and localisation in parallel F-actin bundles of ectoplasmic

Abbreviations: $A B M$, actin-bundling module; $E N G$, electronystagmography; ERG, electroretinography; IRB, Institutional Review Board; $R P$, retinitis pigmentosa 
PKSN32

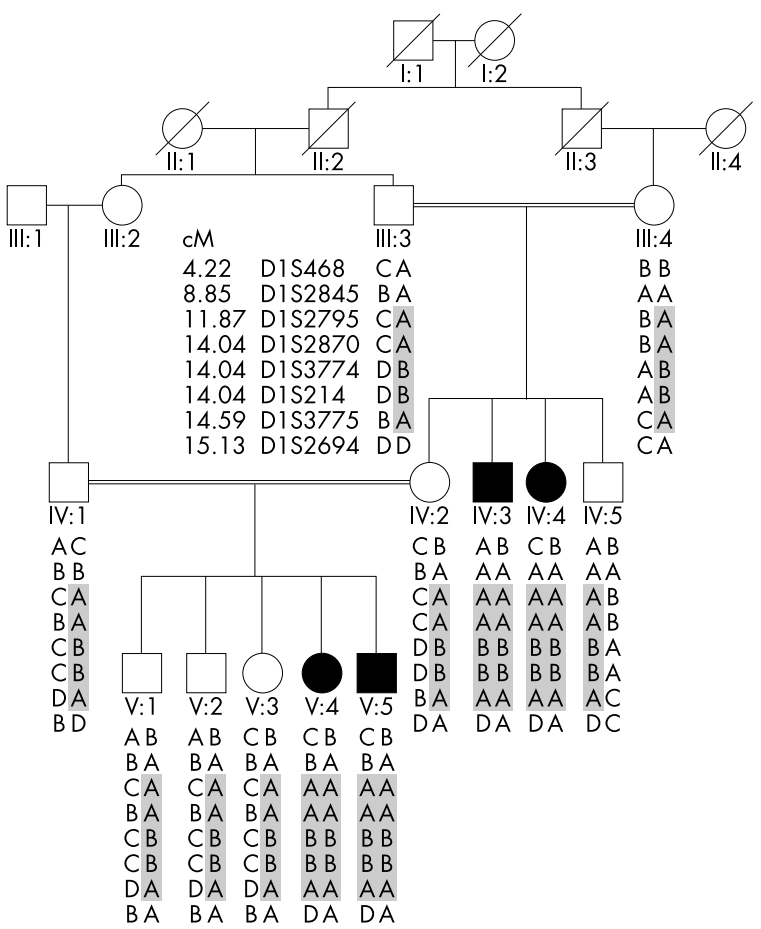

PKSR5A

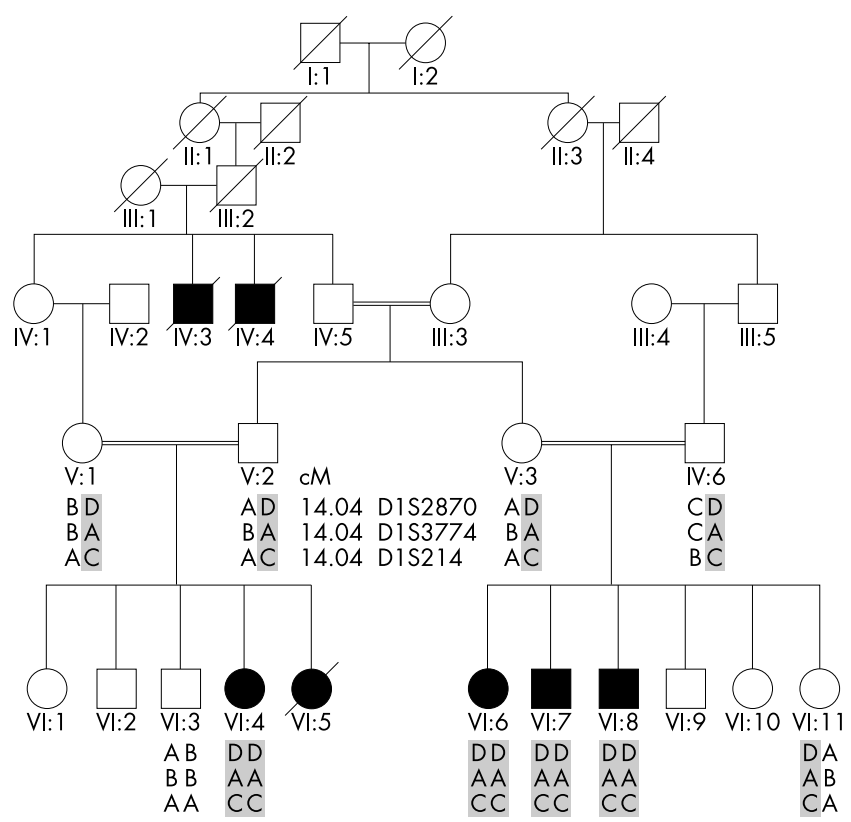

Figure 1 Pedigrees and mapping of DFNB36. Families PKSN32 and PKSR5A are shown with the haplotypes of chromosome 1p36.3 STR markers. The grey shaded haplotypes represent the ancestral chromosome harbouring the ESPN mutation.

specialisation in testes. ${ }^{6}$ Domain prediction algorithms of human espin sequence revealed eight ankyrin-like repeats at the N-terminus, two proline rich regions, a consensus site for ATP or GTP binding (P-loop), which is contained within an actin-binding WH2 motif (amino acids 651-668), and a coiled coil (amino acids 756-831) (figs 2D and 3). There are 66 residues (amino acids $739-804$ ) that show 33\% sequence identity to a domain in forked proteins, which are essential for formation of actin bundles in bristles of Drosophila melanogaster. $^{7}$ Deletion mutagenesis experiments have suggested that espin contains three actin-binding sites (figs $2 \mathrm{D}$ and 3 ). ${ }^{58}$ Two of the actin-binding sites at the Cterminus constitute the ABM (figs 2D and 3) and are important for espin activity. Espn expression constructs lacking either one or both of these actin-binding sites are unable to cross-link actin filaments when transfected into BHK fibroblasts. ${ }^{5}$

The two mutations of ESPN, segregating with hearing loss linked to DFNB36, cause frameshifts in the ESPN translation reading frame. If translated, 1988delAGAG is predicted to

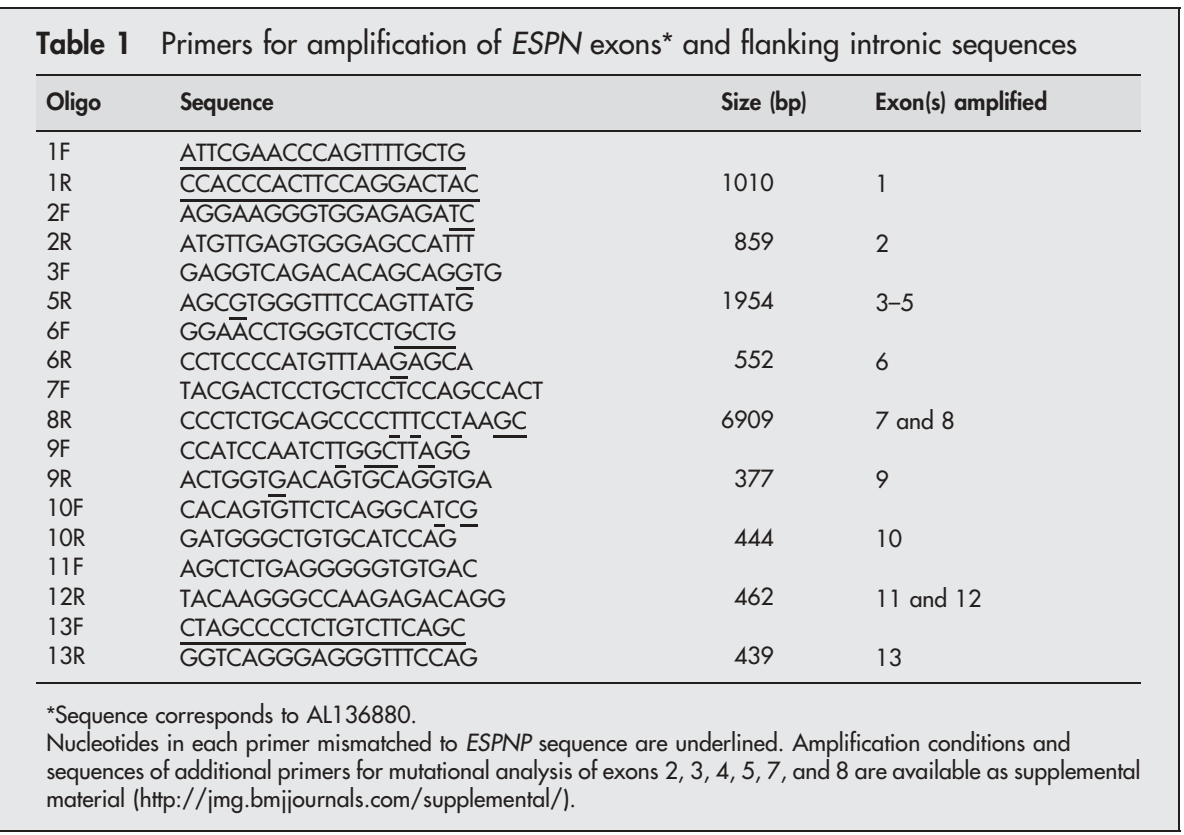


A

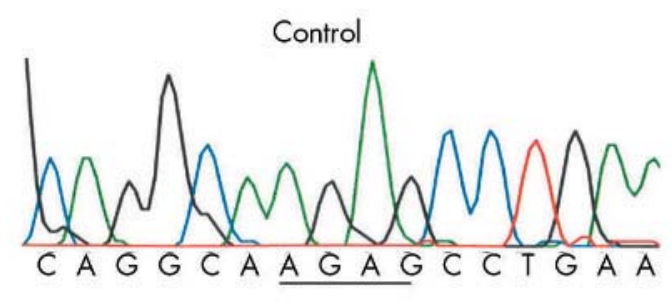

PKSR5A affected (1988delAGAG)

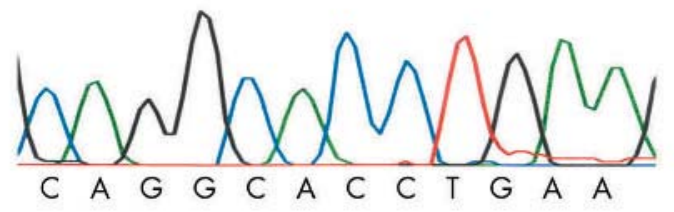

B

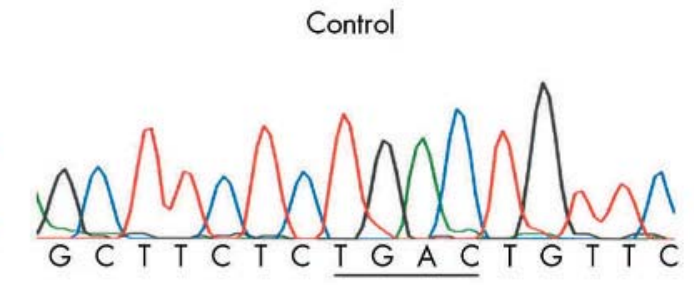

PKSN32 affected (2469delGTCA)

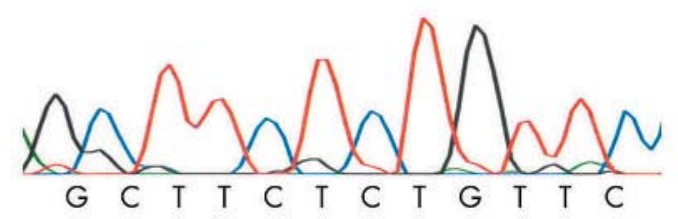

c

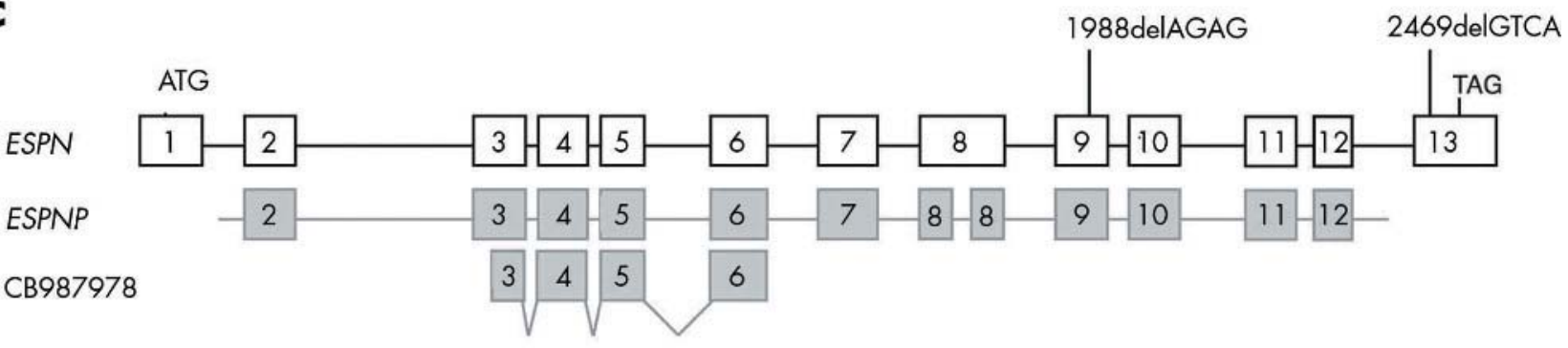

D Ankyrin repeats

$P R 1 \times A B$ PR2

WH2 ABM

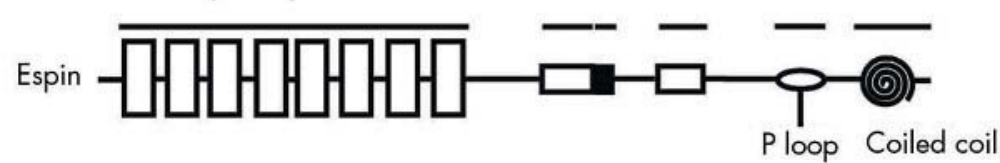

Figure 2 Electropherograms demonstrating ESPN mutations and diagrammatic representation of ESPN and ESPNP. (A) Sequence traces from exon 9 of ESPN for control individual VI:3 and affected individual VI:6 in family PKSR5A, showing the wild type sequence and 1988delAGAG, respectively (underlined in the control trace). (B) Sequence traces from exon 13 of ESPN for a control and affected individual V:4 in family PKSN32, showing 2469delGTCA (underlined). The traces show the reverse complement strand. (C) Exon/intron structure of ESPN (GenBank\# AL136880). Exons are represented as numbered boxes, and introns are indicated by lines joining the boxes (not drawn to scale). The locations of mutations found in the sequencing analysis of ESPN from the affected individuals in the two families are shown (numbered vertical lines). ESPNP, a putative pseudogene, is depicted below ESPN. Exons 1 and 13 of ESPN are absent and exon 8 is interrupted by an intron. There is only one expressed sequence tag (EST) corresponding to ESPNP (GenBank\# CB987978), drawn below ESPNP. (D) Diagrammatic representation of espin depicting ankyrin repeats encoded by exons 1-4 and the proline rich peptides (PR1 and PR2) encoded by exons 7 and 8. $x A B$ is an actin-binding domain and is encoded by exon 7 of ESPN. The WH2 domain (Wiskott Aldrich syndrome homology region 2) containing the P-loop is encoded by exon 9. The coiled coil and other residues important for actin bundling are formed by amino acids encoded by exons 10-13 constituting the actin-bundling module (ABM).

introduce one substituted amino acid after residue 662, followed by a stop codon at nucleotide 1990 and 2469delGTCA is predicted to introduce 27 substituted amino acids after residue $82 \mathrm{l}$, followed by a stop codon at nucleotide 2533 resulting in a truncated 844 amino acid protein with 821 correct and 23 substituted residues. These mutations are presumed to result in loss of function of espin. The resulting mutant proteins are predicted to have either no actin-bundling module, (1988delAGAG), or lack one of the C-terminal actin-binding sites (2469delGTCA), which is necessary for espin activity. ${ }^{5}$ There is evidence for multiple isoforms of Espn in the mouse ${ }^{4569}$ and the same may be true in humans as well. However, the mutations associated with $D F N B 36$ occur in ESPN exons, which are known to be present in all reported isoforms of Espn in mice. ${ }^{4579}$

PCR analysis of human fetal inner ear cDNA revealed expression of ESPN in the inner ear (data not shown). In both the cochlea and the vestibule of the mouse inner ear, espin is localised mostly to the stereocilia of hair cells. ${ }^{4}$ Stereocilia are specialised microvilli projecting from the apical surfaces of inner ear hair cells and are vital for transduction of sound and for detection of linear and angular acceleration. They contain a densely packed core of parallel bundles of actin filaments. ${ }^{10}$ The formation and ordered arrangement of these filaments into bundles requires different actin-binding proteins, which cross-link actin filaments. ${ }^{11}$ Multiple actinbinding proteins such as fimbrin, plastin, and espin, are expressed in the inner ear. ${ }^{11}{ }^{12}$ Espin plays a crucial role in cross-linking parallel actin bundles and varying concentrations of espin may determine the extent of elongation and, consequently, the length of parallel actin bundles. ${ }^{13}$

Espin is absent from the stereocilia of jerker mice, ${ }^{4}$ and consequently by postnatal day 10 the stereocilia are shortened and have reduced stiffness. ${ }^{14}$ Within 3 months of birth, a degenerative process leads to the complete loss of all sensory hair cells in the jerker mouse. ${ }^{4}{ }^{14}$ In addition to espin, 


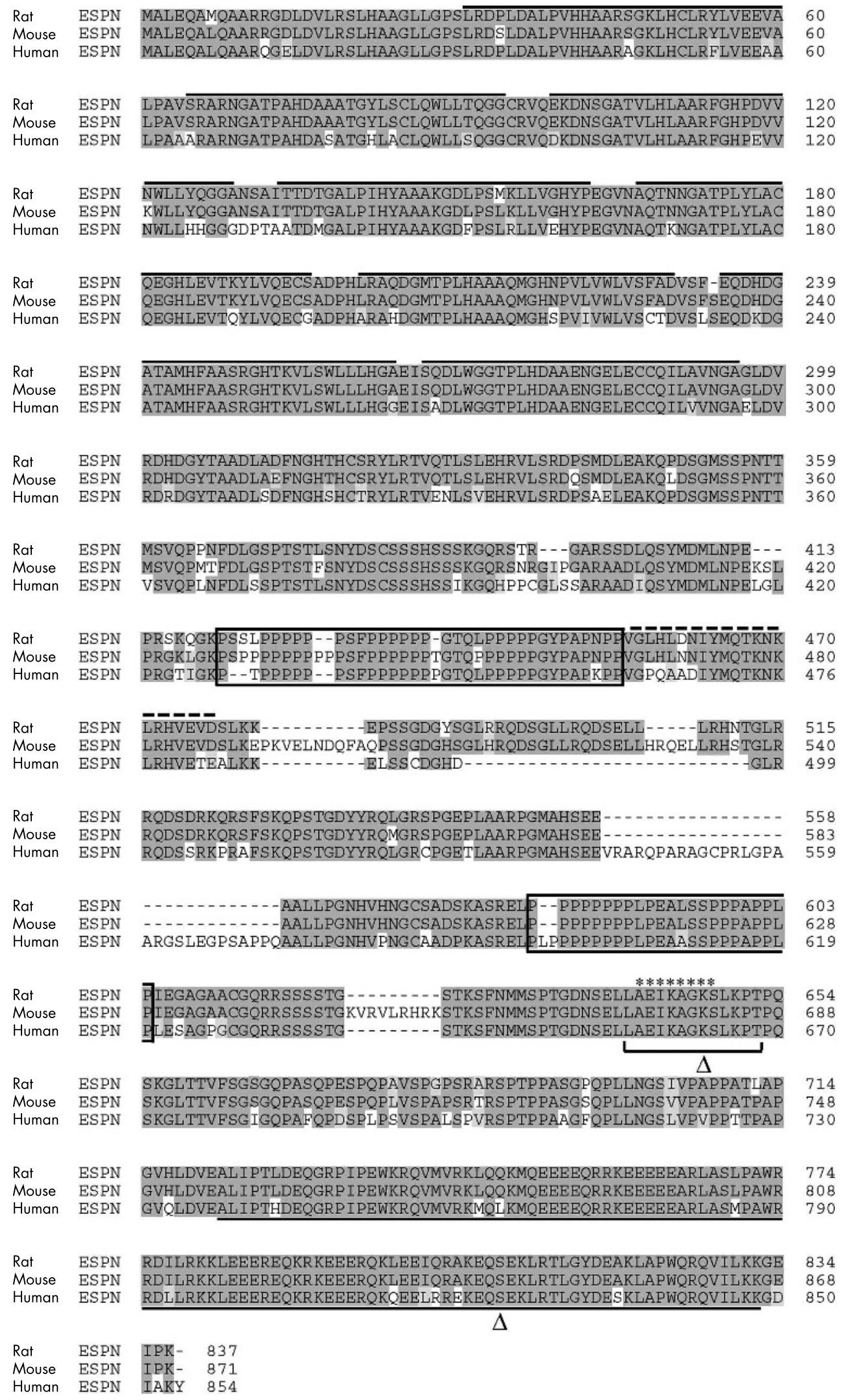

Figure 3 Espin sequence alignment. ClustalW multiple protein alignment of rat, mouse, and human ESPN. Dark shaded residues denote identical amino acids. Light grey shading represents conserved amino acid substitutions and "-" indicates a gap in the alignment. Bars on top of amino acid residues at the $\mathrm{N}$-terminus indicate the location of ankyrin repeats. Two proline rich regions are boxed. A dashed line indicates actin-binding amino acids (xAB). Asterisks mark the amino acids corresponding to the P-loop. Amino acids forming the WH2 domain are indicated by a bracket. Amino acids constituting the actin-bundling module, $A B M, 5$ are underlined. The first amino acid affected by 1988 delAGAG or 2469delGTCA is indicated by " $\Delta$ ". The amino acids shared by mouse and human espin proteins exhibit $83 \%$ identity and $88 \%$ similarity. Human and rat ESPN are $86 \%$ identical and $90 \%$ similar. 
there are many other cytoskeletal proteins that are necessary for the development and maintenance of stereocilia. Mutations of ACTG1 encoding $\gamma$-actin were recently reported to cause progressive hearing loss in humans. ${ }^{15}$ Both $\beta$ - and $\gamma$-actin are present in the stereocilia of auditory hair cells in chicken, ${ }^{16}$ raising the possibility that espin interacts with either $\beta$-actin, $\gamma$-actin, or both in the stereocilia. Moreover, espin has multiple sites for protein-protein interactions, which may serve as a scaffold for assembly of macromolecular complexes important for structure and function of the stereocilia.

Our findings indicate that espin is essential for both hearing and balance in humans. The association of profound deafness and vestibular dysfunction in the absence of other associated phenotypes is unusual, although vestibular dysfunction is often not carefully evaluated or documented for most non-syndromic recessive deafness loci. Vestibular dysfunction has been excluded for 11 DFNB loci: $B 1, B 6$, $B 7 / 11, B 12, B 17, B 18, B 21, B 23, B 26, B 29$, and $B 30^{17}$ (see Homepage Hereditary Hearing Loss Homepage for individual references: http://dnalab-www.uia.ac.be/dnalab/hhh/). Affected individuals with mutations of MYO7A linked to DFNB2 have a vestibular phenotype ${ }^{17}$ comparable to that associated with DFNB36 and affected individuals with mutations of MYO15A also exhibit signs and symptoms of vestibular dysfunction. ${ }^{17}$ Vestibular dysfunction was also reported in a few deaf individuals who have recessive mutations of $M Y O 6 .^{18}$ The abnormal vestibular phenotype associated with ESPN mutations is unusual and will be a useful clinical marker for refining the differential diagnosis of non-syndromic deafness.

\section{ACKNOWLEDGEMENTS}

We are grateful to the families for their participation and the staff of Children's Hospital, Lahore, Pakistan, for their cooperation and help in providing facilities for ENG examinations. RNA for cDNA synthesis was received from C C Morton, who obtained human fetal ear tissue under IRB approval by the Human Research Committee of Brigham and Women's Hospital. We thank James Bartles, Inna Belyantseva, Erich Boger, Rafael Caruso, Dennis Drayna, Robert Morell, Barbara Ploplis, and Susan Sullivan for their comments and expertise.

\section{Authors' affiliations}

S Naz, S Riazuddin, E R Wilcox, T B Friedman, Section on Human Genetics, LMG, NIDCD, NIH, Rockville, MD 20850, USA

A J Griffith, Section on Gene Structure and Function, LMG, NIDCD, NIH, Rockville, MD, USA

A J Griffith, Hearing Section, NIDCD, NIH, Rockville, MD, USA

L L Hampton, J F Battey Jr, Section on G-protein Coupled Receptors, NINDS, NIH, Bethesda, MD, USA

S N Khan, S Riazuddin, National Center of Excellence in Molecular Biology, University of the Punjab, Lahore, Pakistan

Part of the study carried out in Pakistan was supported by a grant from the Higher Education Commission in Islamabad. Intramural funds from the National Institute on Deafness and Other Communication Disorders
(Z01 DC000035-07, Z01 DC000064-03, Z01 DC000039-07) supported this work.

Conflict of interest: none declared.

GenBank accession numbers: mouse Espn AF239886, rat Espn NM_019622.1, human ESPN AL136880, ESPNP (LOC284729) ALO35288; ESPN genomic sequence AL031848 and AL158217; genomic sequence for ESPNP AL021920; EST for ESPNP CB987978.

Correspondence to: DrT B Friedman, Laboratory of Molecular Genetics, NIDCD, NIH, Rockville, MD 20850, USA; friedman@nidcd.nih.gov

Revised version received 8 March 2004

Accepted for publication 10 March 2004

\section{REFERENCES}

1 Morton NE. Genetic epidemiology of hearing impairment. Ann NY Acad Sci $1991 ; 630: 16-31$.

2 Friedman TB, Griffith AJ. Human nonsyndromic sensorineural deafness. Annu Rev Genomics Hum Genet 2003;4:341-402.

3 Smith RJ, Berlin CI, Hejtmancik JF, Keats BJ, Kimberling WJ, Lewis RA, Moller CG, Pelias MZ, Tranebjaerg L. Clinical diagnosis of the Usher syndromes. Am J Med Genet 1994:50:32-8.

4 Zheng L, Sekerkova G, Vranich K, Tilney LG, Mugnaini E, Bartles JR. The deaf jerker mouse has a mutation in the gene encoding the espin actin-bundling proteins of hair cell stereocilia and lacks espins. Cell 2000;102:377-85.

5 Bartles JR, Zheng L, Li A, Wierda A, Chen B. Small espin: a third actinbundling protein and potential forked protein ortholog in brush border microvilli. J Cell Biol 1998;143:107-19.

6 Bartles JR, Wierda A, Zheng L. Identification and characterization of espin, an actin-binding protein localized to the F-actin-rich junctional plaques of Sertoli cell ectoplasmic specializations. J Cell Sci 1996;109:1229-39.

7 Petersen NS, Lankenau DH, Mitchell HK, Young P, Corces VG. Forked proteins are components of fiber bundles present in developing bristles of Drosophila melanogaster. Genetics 1994;136:173-82.

8 Chen B, Li A, Wang D, Wang M, Zheng L, Bartles JR. Espin contains an additional actin-binding site in its $\mathrm{N}$ terminus and is a major actin-bundling protein of the Sertoli cell-spermatid ectoplasmic specialization junctional plaque. Mol Biol Cell 1999; 10:4327-39.

9 Sekerkova G, Loomis PA, Changyaleket B, Zheng L, Eytan R, Chen B, Mugnaini E, Bartles JR. Novel espin actin-bundling proteins are localized to Purkinje cell dendritic spines and bind the Src homology 3 adapter protein insulin receptor substrate p53. J Neurosci 2003;23:1310-19.

10 Tilney LG, Tilney MS, DeRosier DJ. Actin filaments, stereocilia, and hair cells: how cells count and measure. Annu Rev Cell Biol 1992;8:257-74.

11 Bartles JR. Parallel actin bundles and their multiple actin-bundling proteins. Curr Opin Cell Biol 2000;12:72-8.

12 Daudet N, Lebart MC. Transient expression of the t-isoform of plastins/fimbrin in the stereocilia of developing auditory hair cells. Cell Motil Cytoskeleton 2002; 53:326-36.

13 Loomis PA, Zheng L, Sekerkova G, Changyaleket B, Mugnaini E, Bartles JR. Espin cross-links cause the elongation of microvillus-type parallel actin bundles in vivo. J Cell Biol 2003;163:1045-55.

14 Sjostrom B, Anniko M. Genetically induced inner ear degeneration. A structural and functional study. Acta Otolaryngol Suppl 1992;493:141-46.

15 Zhu M, Yang T, Wei S, DeWan AT, Morell RJ, Elfenbein JL, Fisher RA, Leal SM, Smith RJ, Friderici KH. Mutations in the gamma-actin gene (ACTG1) are associated with dominant progressive deafness (DFNA20/26). Am J Hum Genet 2003;73:1082-91.

16 Hofer D, Ness W, Drenckhahn D. Sorting of actin isoforms in chicken auditory hair cells. J Cell Sci 1997;110:765-70.

17 Cremers CWRJ, Smith RJH, eds. Genetic hearing impairment, its clinical presentations. Adv Otorhinolaryngol 2002;61:113-56.

18 Ahmed ZM, Morell RJ, Riazuddin S, Gropman A, Shaukat S, Ahmad MM, Mohiddin SA, Fananapazir L, Caruso RC, Husnain T, Khan SN, Griffith AJ Riazuddin S, Friedman TB, Wilcox ER. Mutations of MYO6 are associated with recessive deafness, DFNB37. Am J Hum Genet 2003;72:1315-22. 